\title{
Muon-spin rotation measurements of the penetration depth of the $\mathrm{Mo}_{3} \mathrm{Sb}_{7}$ superconductor
}

\author{
R. Khasanov, ${ }^{1, *}$ P. W. Klamut, ${ }^{2}$ A. Shengelaya,${ }^{3}$ Z. Bukowski, ${ }^{2,4}$ I. M. Savić, ${ }^{5}$ C. Baines, ${ }^{1}$ and H. Keller ${ }^{6}$ \\ ${ }^{1}$ Laboratory for Muon Spin Spectroscopy, Paul Scherrer Institut, CH-5232 Villigen PSI, Switzerland \\ ${ }^{2}$ Institute of Low Temperature and Structure Research of the Polish Academy of Sciences, Okólna 2, 50-422 Wroctaw, Poland \\ ${ }^{3}$ Physics Institute of Tbilisi State University, Chavchavadze 3, GE-0128 Tbilisi, Georgia \\ ${ }^{4}$ Laboratory for Solid State Physics, ETH Zürich, CH-8093 Zürich, Switzerland \\ ${ }^{5}$ Faculty of Physics, University of Belgrade, 11001 Belgrade, Serbia \\ ${ }^{6}$ Physik-Institut der Universität Zürich, Winterthurerstrasse 190, CH-8057 Zürich, Switzerland
}

(Received 9 April 2008; revised manuscript received 27 May 2008; published 2 July 2008)

\begin{abstract}
Measurements of the magnetic-field penetration depth $\lambda$ in superconductor $\mathrm{Mo}_{3} \mathrm{Sb}_{7}\left(T_{c} \simeq 2.1 \mathrm{~K}\right)$ were carried out by means of muon-spin rotation. The absolute values of $\lambda$, the Ginzburg-Landau parameter $\kappa$, and the first $H_{c 1}$ and the second $H_{c 2}$ critical fields at $T=0$ are $\lambda(0)=720(100) \mathrm{nm}, \kappa(0)=55(10), \mu_{0} H_{c 1}(0)$ $=1.8(3) \mathrm{mT}$, and $\mu_{0} H_{c 2}(0)=1.9(2) \mathrm{T}$. The zero-temperature value of the superconducting energy gap $\Delta(0)$ was found to be $0.35(1) \mathrm{meV}$, corresponding to the ratio $2 \Delta(0) / k_{B} T_{c}=3.83(10)$. At low temperatures $\lambda^{-2}(T)$ saturates and becomes constant below $T \simeq 0.3 T_{c}$, in agreement with what is expected for $s$-wave BCS superconductors. Our results suggest that $\mathrm{Mo}_{3} \mathrm{Sb}_{7}$ is a superconductor with the single isotropic energy gap.
\end{abstract}

DOI: 10.1103/PhysRevB.78.014502 PACS number(s): 74.70.Ad, 74.25.Op, 74.25.Ha, 76.75.+i

Recently, the attention was devoted to $\mathrm{Mo}_{3} \mathrm{Sb}_{7}$. This compound was originally discovered more than 40 years ago ${ }^{1,2}$ and only recently was found to become a type-II superconductor with the transition temperature $T_{c} \simeq 2.1 \mathrm{~K}^{3}$ The properties of $\mathrm{Mo}_{3} \mathrm{Sb}_{7}$ in a superconducting state are rather unusual. Specific heat, resistivity, and magneticsusceptibility experiments of Candolfi et al. ${ }^{4}$ suggest that $\mathrm{Mo}_{3} \mathrm{Sb}_{7}$ can be classified as a coexistent superconductorspin-fluctuating system. As discussed in Ref. 4, factoring in the effect of spin fluctuations leads to renormalized values of the electron-phonon coupling constant and the Coulomb pseudopotential, which, being substituted to the McMillan expression, lead to $T_{c}$ between 1.4 and $2.0 \mathrm{~K}$. This is substantially closer to the experimentally observed $T_{c} \simeq 2.1 \mathrm{~K}$ than $T_{c} \approx 10 \mathrm{~K}$, which would be obtained without taking into account the effect of spin fluctuations. ${ }^{4}$

There is currently no agreement on the symmetry of the order parameter of $\mathrm{Mo}_{3} \mathrm{Sb}_{7}$. The recent specific-heat experiments of Candolfi et al. ${ }^{5}$ suggest that the order parameter in $\mathrm{Mo}_{3} \mathrm{Sb}_{7}$ is of conventional $s$-wave symmetry. Tran et al., ${ }^{6}$ based again on the results of specific-heat measurements, reported the presence of two isotropic $s$-wave gaps with $2 \Delta_{1}(0) / k_{B} T_{c}=4.0$ and $2 \Delta_{2}(0) / k_{B} T_{c}=2.5[\Delta(0)$ is the zerotemperature value of the superconducting energy gap]. In contrast, Andreev-reflection measurements of Dmitriev et $a .^{7-9}$ reveal that the superconducting gap is highly anisotropic. The ratio of the maximum to the minimum gap was estimated to be $\Delta_{\max } / \Delta_{\min } \simeq 40$ and $s+g$-wave symmetry of the order parameter was proposed in a qualitative analysis. ${ }^{8,9}$

The symmetry of the superconducting order parameter can be probed by measurements of the magnetic penetration depth $\lambda$. A fully gaped, isotropic pairing state produces a thermally activated behavior, leading to an almost constant value of the superfluid density $\rho_{s} \propto \lambda^{-2}$ for $T \leqq 0.3 T_{c}{ }^{10,11}$ Presence of nodes in the gap leads to a continuum of lowlaying excitations, which result in a linear $\lambda^{-2}(T)$ at low temperatures. ${ }^{12,13}$ In two-gap superconductors with highly different gap to $T_{c}$ ratios, the inflection point in $\lambda^{-2}(T)$ is generally present. ${ }^{14-16}$
In this paper, we report the study of the magnetic field penetration depth in superconductor $\mathrm{Mo}_{3} \mathrm{Sb}_{7}$ by means of muon-spin rotation. Measurements were performed down to $20 \mathrm{mK}$ in a series of fields ranging from 0.02 to $0.2 \mathrm{~T}$. Our results are well explained assuming conventional superconductivity with the single isotropic energy gap, in agreement with the recent specific-heat experiments of Candolfi et al. ${ }^{5}$ The zero-temperature value of the gap was found to be $\Delta(0)=0.35(1) \mathrm{meV}$, corresponding to the ratio $2 \Delta(0) / k_{B} T_{c}$ $=3.83(10)$.

The $\mathrm{Mo}_{3} \mathrm{Sb}_{7}$ single-crystal samples were grown through peritectic reaction between Mo metal and liquid $\mathrm{Sb}^{3}$ The transverse-field muon-spin rotation $(\mathrm{TF}-\mu \mathrm{SR})$ experiments were performed at the $\pi \mathrm{M} 3$ beam line at Paul Scherrer Institute (Villigen, Switzerland). For our experiments the ensemble of some sub- and millimeter size single crystals were mounted onto the silver plate to cover the area of approximately $50 \mathrm{~mm}^{2}$. The silver sample holder was used because it gives a nonrelaxing muon signal and, hence, only contributes as temperature-independent constant background. The crystals were oriented so that the magnetic field was preferably applied along the 001 crystallographic direction. The $\mathrm{Mo}_{3} \mathrm{Sb}_{7}$ samples were field cooled from above $T_{c}$ down to $\simeq 20 \mathrm{mK}$ in magnetic fields ranging from 0.02 to $0.2 \mathrm{~T}$. A full temperature scan (from 0.02 up to $2.5 \mathrm{~K}$ ) was performed in a field of $\mu_{0} H=0.02 \mathrm{~T}$.

Figure 1 shows the muon-spin precession signals in $\mu_{0} H=0.02 \mathrm{~T}$ above $(T=2.2 \mathrm{~K})$ and below $(T=0.05 \mathrm{~K})$ the superconducting transition temperature of $\mathrm{Mo}_{3} \mathrm{Sb}_{7}$. The difference in the relaxation rate above and below $T_{c}$ is due to the well-known fact that type-II superconductors exhibit a flux-line lattice, leading to spatial inhomogeneity of the magnetic induction. As is shown by Brandt, ${ }^{17,18}$ the second moment of this inhomogeneous field distribution is related to the magnetic-field penetration depth $\lambda$ in terms of $\left\langle\Delta B^{2}\right\rangle \propto \sigma_{s c}^{2} \propto \lambda^{-4}$.

The analysis of TF- $\mu \mathrm{SR}$ data was carried out in the time domain by using the following functional form: ${ }^{19}$ 


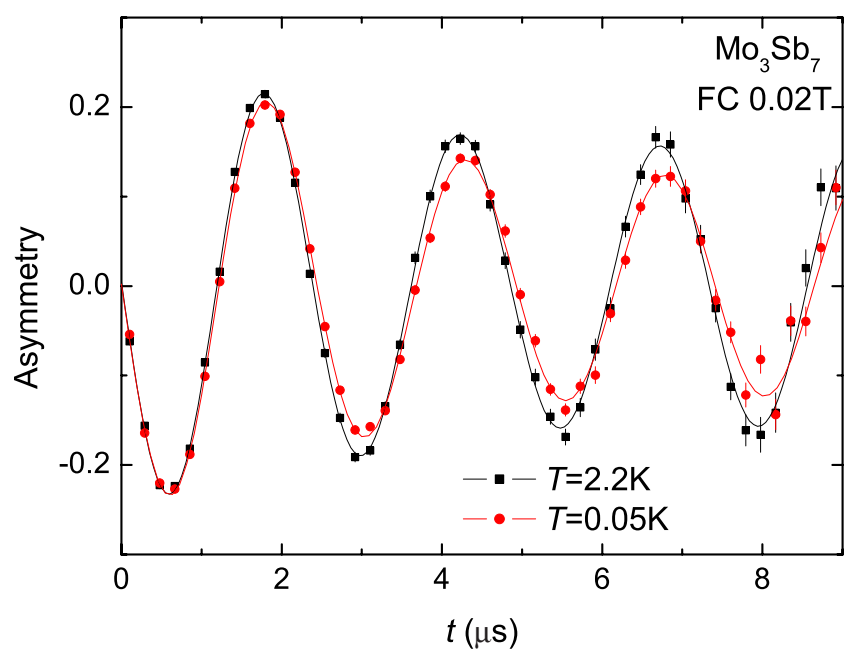

FIG. 1. (Color online) Transverse-field muon-spin precession signals from $\mathrm{Mo}_{3} \mathrm{Sb}_{7}$ obtained in $\mu_{0} H=0.02 \mathrm{~T}$ above the transition temperature $[T=2.2 \mathrm{~K}$ (black squares)] and after field cooling the sample below $T_{c}[T=0.05 \mathrm{~K}$ (red circles)]. The solid lines correspond to the fit by means of Eq. (1). For visualization purpose the apparent precession frequencies are modified from the actual precession frequencies by the use of a rotating reference frame.

$$
\begin{aligned}
A(t)= & A_{0} \exp \left[-\frac{\sigma_{n m}^{2}+\sigma_{s c}^{2}}{2} t^{2}\right] \cos \left(\gamma_{\mu} B_{\mathrm{int}} t+\phi\right) \\
& +A_{b g} \cos \left(\gamma_{\mu} B_{\mathrm{ext}} t+\phi\right),
\end{aligned}
$$

where the first term denotes the contribution from the $\mathrm{Mo}_{3} \mathrm{Sb}_{7}$ sample, and the second term is the background contribution from the $\mathrm{Ag}$ sample holder. $A_{0}$ and $A_{b g}$ are the initial asymmetries arising from the sample and the background. $B_{\text {int }}$ and $B_{\text {ext }}$ are the internal filed inside the sample and the applied external field seen in the Ag backing plate. $\gamma_{\mu}=2 \pi \times 135.5342 \mathrm{MHz} / \mathrm{T}$ is the muon gyromagnetic ratio, and $\phi$ is the initial phase. $\sigma_{s c}$ and $\sigma_{n m}$ are the muon-spin relaxation rates caused by the nuclear moments and the additional component appearing below $T_{c}$ due to nonuniform field distribution in the superconductor in the mixed state.

The analysis was carried out as follows. First, the muon-time spectra were fitted by means of Eq. (1) with all the parameters free. Then, the ratio $A_{0} / A_{b g}=0.975$ was obtained as the mean value in the temperature interval of $0.02 \mathrm{~K} \leq T \leq 1.5 \mathrm{~K}$. After that, by keeping the ratio $A_{b g} / A_{0}$ fixed, the nuclear-moment contribution $\sigma_{n m}$ was estimated by analyzing the experimental data above $2.1 \mathrm{~K}$, (i.e., at $T>T_{c}$, where $\left.\sigma_{s c}=0\right)$. The final analysis was made with $A_{0} / A_{b g}, \sigma_{n m}$, and $B_{\text {ext }}$ as fixed, and $B_{\text {int }}$ and $\sigma_{s c}$ as free parameters. This allows us to reduce the number of the fitting parameters and, as a consequence, to increase the accuracy in determination of $\sigma_{s c}$.

Within Bardeen-Cooper-Schrieffer (BCS) theory the zerotemperature magnetic penetration depth $[\lambda(0)]$ of isotropic superconductor does not depend on the magnetic field. Contrary, the nonlocal and the nonlinear response of the superconductor with nodes in the gap to the magnetic field, as well as the faster suppression of the contribution of the smaller gap to the total superfluid density in a case of two-gap su- perconductor, lead to the fact that $\lambda(0)$, evaluated from $\mu \mathrm{SR}$ experiments, is magnetic field dependent and it increases with increasing field. ${ }^{20,21}$ Such behavior was observed in various hole-doped cuprates, ${ }^{13,22,23}$ and the double-gap $\mathrm{NbSe}_{2}$ (Refs. 22 and 24) and $\mathrm{MgB}_{2}$ superconductors. ${ }^{25,26}$

Consideration of the ideal vortex lattice (VL) of an isotropic $s$-wave superconductor within Ginsburg-Landau approach leads to the following expression for the magneticfield dependence of the second moment of the magnetic-field distribution: ${ }^{18}$

$$
\sigma_{s c}\left[\mu \mathrm{s}^{-1}\right]=a \times\left(1-B / B_{c 2}\right)\left[1+1.21\left(1-\sqrt{B / B_{c 2}}\right)^{3}\right] \lambda^{-2}[\mathrm{~nm}] .
$$

Here $B$ is the magnetic induction, which for applied fields in the region $H_{c 1} \ll H_{\text {app }} \ll H_{c 2}$ is $B \simeq \mu_{0} H_{\text {app }}, a$ is the coefficient depending on the symmetry of the VL $\left[a=4.83 \times 10^{4}\right.$ for triangular VL (Refs. 10 and 18) and, as is shown below, $a$ $=5.07 \times 10^{4}$ for rectangular VL], $H_{c 1}$ is the first critical field, and $B_{c 2}=\mu_{0} H_{c 2}$ is the upper critical field. Equation (2) accounts for reduction of $\sigma_{s c}$ due to stronger overlapping of vortices by their cores with increasing field. According to the calculations of Brandt, ${ }^{18}$ Eq. (2) describes with less than $5 \%$ error the field variation of $\sigma_{s c}$ for an ideal triangular VL, and it holds for type-II superconductors with the value of the Ginzburg-Landau parameter $\kappa=\lambda / \xi \geq 5$ ( $\xi$ is the coherence length) in the range of fields $0.25 / \kappa^{1.3} \leqslant B / B_{c 2} \leq 1$.

Satisfactory fit of Eq. (2) to the experimental data would suggest that there is no significant change of the penetration depth in the range of magnetic fields of the experiment. We performed an analysis of the magnetic-field dependence of the muon depolarization rate $\sigma_{s c}$ measured at $T=20 \mathrm{mK}$ (see Fig. 2), which is a good approximation of $\sigma_{s c}(T=0, B)$. The solid line in Fig. 2 corresponds to the fit of experimental $\sigma_{s c}(B)$ by means of Eq. (2) for a case of triangular VL. The fit yields $\lambda(0)=720(25) \mathrm{nm}$ and $B_{c 2}(0)=1.9(2)$ T. A good agreement between the experiment and the theory (see Fig. 2) allows us to conclude that $\sigma_{s c}(T=0, B)$ can be consistently fitted within the behavior expected for conventional isotropic superconductor. For comparison in the inset of Fig. 2 we plot the dependence of the inverse-squared penetration depth normalized to its value at $B=0$ on the reduced field $b=B / B_{c 2}$ for $\mathrm{Mo}_{3} \mathrm{Sb}_{7}$ studied here, the two-gap superconductor $\mathrm{MgB}_{2}$ from Ref. 26, and the hole-doped cuprate superconductor $\mathrm{YBa}_{2} \mathrm{Cu}_{3} \mathrm{O}_{6.95}$ from Ref. 22. It is seen that in a case of $\mathrm{MgB}_{2}$ and $\mathrm{YBa}_{2} \mathrm{Cu}_{3} \mathrm{O}_{6.95}, \lambda^{-2}$ evaluated from muon experiments is field dependent and decreases rather strongly with increasing field.

The absolute error in $\lambda(0)$, estimated from the fit of $\sigma_{s c}(B)$ by means of Eq. (2), is $\simeq 3.5 \%$. There are, however, other sources of uncertainties, which could also affect $\lambda(0)$ and, consequently, increase the error: (i) The use of Eq. (2) to extract $\lambda^{-2}$ from $\sigma_{s c}(B)$ requires that the VL is well ordered and it is triangular, as opposed to disordered or rectangular VL. (ii) The fitting function Eq. (1) assumes that the contribution from the sample is described by a single line of Gaussian shape, as opposed to an asymmetric magnetic-field distribution $P(B)$ generally observed in good quality single crystals. $^{22}$ (iii) The background signal due to the Ag backing 


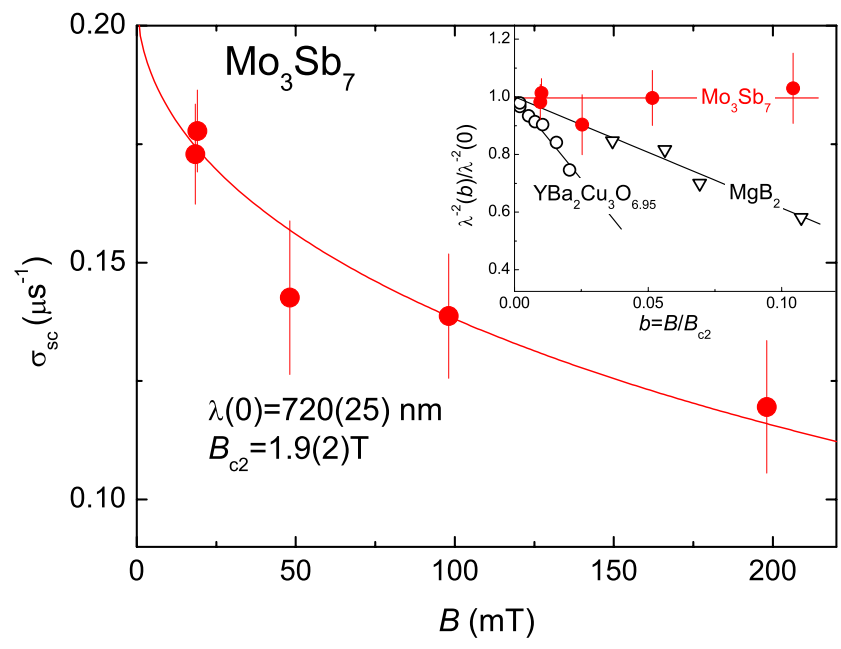

FIG. 2. (Color online) Field dependence of the $\mu$ SR depolarization rate $\sigma_{s c}$ measured at $T=20 \mathrm{mK}$ in $\mathrm{Mo}_{3} \mathrm{Sb}_{7}$ sample. The solid line corresponds to a fit of Eq. (2) for a case of triangular VL to the experimental data with the parameters shown in the figure. The inset shows the dependence of $\lambda^{-2}$ normalized to its value at $B=0$ on the reduced magnetic field $b=B / B_{c 2}$ for $\mathrm{Mo}_{3} \mathrm{Sb}_{7}$, the two-gap superconductor $\mathrm{MgB}_{2}$ from Ref. 26, and the hole-doped cuprate superconductor $\mathrm{YBa}_{2} \mathrm{Cu}_{3} \mathrm{O}_{6.95}$ from Ref. 22 .

plate was assumed to have a temperature-independent frequency and is nonrelaxing at all $T$. However, when the sample goes superconducting it excludes some field, even in the mixed state. These small fields are very inhomogeneous and could cause a shift of the background signal to higher fields and an increase in a slow relaxation. In the following we are going to discuss in detail the sources (i) and (ii). We believe that the source (iii) does not play a substantial role here since even at a $T=20 \mathrm{mK}, B_{\text {int }}$ is only $0.16 \mathrm{mT}$ smaller than the applied field, and $B_{\text {ext }}$ is constant in the whole temperature range (from $20 \mathrm{mK}$ up to $T_{c}$ ). It should be mentioned, however, that experience with more robust superconductors has shown that even though the background field does not usually change perceptibly, the excluded flux can affect the background relaxation rate.

To account for possible random deviations of the flux core positions from their ideal ones (VL disorder) and for broad- ening of $\mu \mathrm{SR}$ spectra due to nuclear depolarization, the field distribution of an ideal VL $\left[P_{i d}(B)\right]$ is, generally, convoluted with a Gaussian distribution in terms of ${ }^{27-29}$

$$
P(B)=\frac{\gamma_{\mu}}{\sqrt{2 \pi} \sigma_{g}} \int \exp \left[-\frac{\gamma_{\mu}^{2}}{2}\left(\frac{B-B^{\prime}}{\sigma_{g}}\right)^{2}\right] P_{i d}\left(B^{\prime}\right) d B^{\prime} .
$$

Here $\sigma_{g}=\sqrt{\sigma_{n m}^{2}+\sigma_{B}^{2}}$ and $\sigma_{B}$ is the contribution to the Gaussian broadening of $P_{i d}(B)$ due to VL disorder. The theoretical $P(B)$ profiles for triangular VL and various $\sigma_{g}$ 's are shown in Fig. 3(a). It is obvious that both the nuclear moment contribution and the VL disorder broaden the $P(B)$ profiles, thus requiring that the total second moment of $\mu$ SR line needs to be the sum of three components: ${ }^{29} \sigma_{s c}^{2}+\sigma_{n m}^{2}+\sigma_{B}^{2}$. This implies that neglecting the VL disorder leads to an overestimation of $\sigma_{s c}$ and, as a consequence, to an underestimation of $\lambda$. On the other hand, $\sigma_{s c}$, obtained from the fit of asymmetric $P(B)$ line by using symmetric Gaussian function [see Eq. (1)], becomes underestimated. ${ }^{22}$ In a case of $\mathrm{Mo}_{3} \mathrm{Sb}_{7}$ the main contribution to $\sigma_{g}$ comes, most probably, from the nuclear-moment term $\sigma_{n m} \simeq 0.178 \mu \mathrm{s}^{-1}$, which is comparable with $\sigma_{s c}$ in the whole field range, rather than from $\sigma_{B}$, which for good quality single crystals corresponds, typically, to $10-20 \%$ of $\sigma_{s c}$ (see, e.g., Ref. 22 and references therein). Such big $\sigma_{n m}$ also leads to the fact that the shape of $P(B)$ is close to the Gaussian one, as is demonstrated by the solid blue line in Fig. 3(a).

Dependences of $\sigma_{s c}$ on the reduced field $b=B / B_{c 2}$ for triangular and rectangular VLs, obtained by using numerical calculations of Brandt, ${ }^{18}$ are shown in Fig. 3(b). Solid lines correspond to Eq. (2) with $a=4.83 \times 10^{4}$ (red line) and $5.07 \times 10^{4}$ (blue line). As is seen, in the case of rectangular VL, $\sigma_{s c}(b)$ can still be satisfactorily described by means of Eq. (2) with the only 5\% bigger value of the coefficient $a$. This implies that the uncertainty related to different VL symmetries result in $\simeq 2.5 \%$ additional error in $\lambda$. To summarize, by taking into account the above presented arguments, we believe that the true overall uncertainty in the absolute value of $\lambda(0)$ is about $10-15 \%$, and $\lambda(0)=720(100) \mathrm{nm}$.

The zero-temperature value of the superconducting coherence length $\xi(0)$ may be estimated from $B_{c 2}(0)$ as $\xi(0)$ $=\left[\Phi_{0} / 2 \pi B_{c 2}(0)\right]^{0.5}$, which results in $\xi(0)=13(1) \mathrm{nm}\left(\Phi_{0}\right.$ is

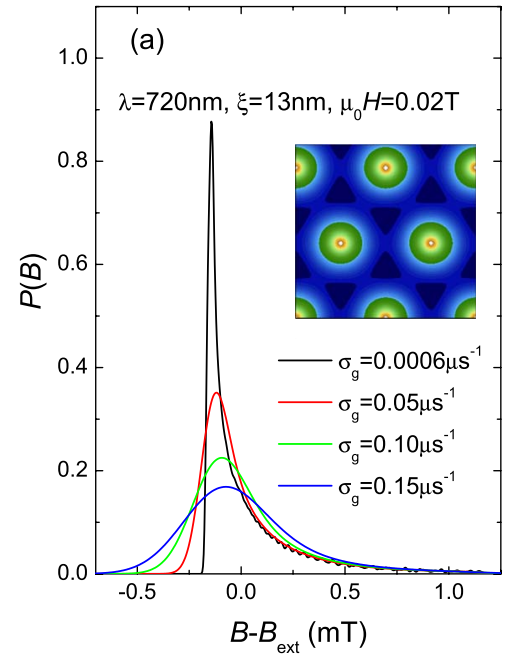

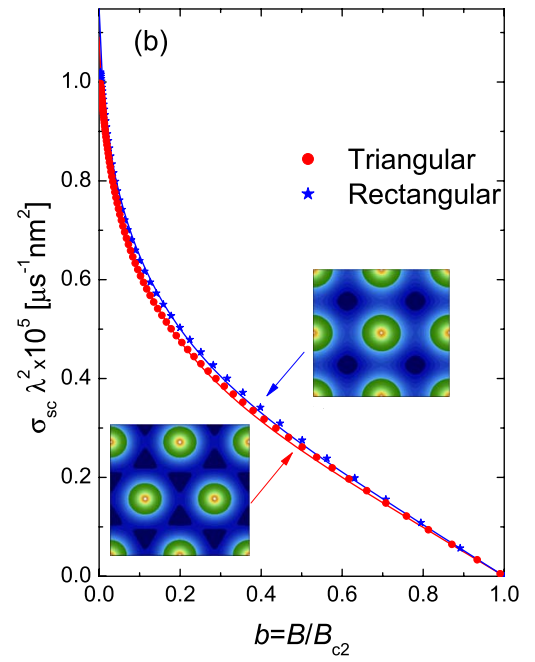

$014502-3$
FIG. 3. (Color online) (a) Theoretical $P(B)$ profiles for triangular VL $(\lambda=720 \mathrm{~nm}, \xi$ $=13 \mathrm{~nm}$, and $\left.\mu_{0} H=0.02 \mathrm{~T}\right)$ calculated for $\sigma_{g}$ $=0.0006,0.05,0.1$, and $0.15 \mu \mathrm{s}^{-1}$. (b) Dependence of the product $\sigma_{s c} \times \lambda^{-2}$ on the reduced field $b=B / B_{c 2}$ for the triangular (circles) and the rectangular (stars) VLs. Calculations were made by using the numerical approach of Brandt ${ }^{18}$ for $\kappa=100$. The solid lines are obtained by means of Eq. (2) with $a=4.83 \cdot 10^{4}$ for triangular and $a$ $=5.07 \cdot 10^{4}$ for rectangular VLs, respectively. The insets are the contour plots of field variation within triangular and rectangular VLs. 


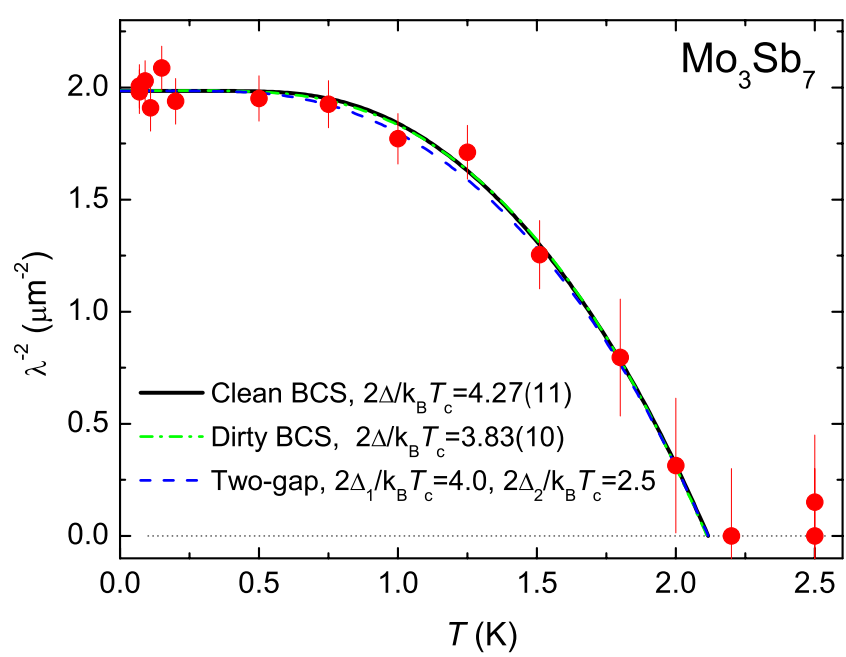

FIG. 4. (Color online) Temperature dependence of $\lambda^{-2}$ of $\mathrm{Mo}_{3} \mathrm{Sb}_{7}$ reconstructed from $\sigma_{s c}(T)$ measured in $\mu_{0} H=0.02 \mathrm{~T}$. The black solid and the dashed-dotted blue lines are the fits by means of clean and dirty weak-coupling BCS models. The blue dashed line represents $\lambda^{-2}(T)$ calculated by using two-gap alpha model ${ }^{14,15}$ with the parameters from Ref. 6 . See text for details.

the magnetic flux quantum). Using the values of $\lambda(0)$ and $\xi(0)$, the zero-temperature value of the Ginzburg-Landau parameter is $\kappa(0)=\lambda(0) / \xi(0)=55(10)$. The first critical field can also be calculated by means of Eq. (4) from Ref. 18 as $\mu_{0} H_{c 1}(0)=1.8(3) \mathrm{mT}$. Note that the zero-temperature values of $\lambda(0), \xi(0), \kappa(0)$, and the first and the second critical fields are in agreement with those reported in the literature. .,5-7,9 $^{3}$

As a next step we are going to analyze the temperature dependence of the magnetic penetration depth. $\lambda^{-2}(T)$, presented in Fig. 4, was reconstructed from $\sigma_{s c}(T)$ measured in a field of $0.02 \mathrm{~T}$ by using Eq. (2). $B_{c 2}(T)$, needed for such reconstruction, was assumed to follow the WerthamerHelfand-Hohenberg prediction, ${ }^{30}$ in agreement with the results of Ref. 5. It is worth mentioning here that the temperature dependence of $\lambda^{-2}$ is much less affected by the above described uncertainties than the absolute $\lambda(0)$ value. Indeed, both $\sigma_{B}$ [see Eq. (3), and Refs. 28 and 29] and the relaxation related to the excluded flux increase with decreasing $T$ in much the same way as $\sigma_{s c}$, which causes mainly a correction to the coefficient $a$ in Eq. (2), while $\sigma_{n m}$ is $T$ independent.

Figure 4 implies that below $0.7 \mathrm{~K}, \lambda^{-2}$ is temperature independent, as expected for the superconductor with fully gaped states. The experimental $\lambda^{-2}(T)$ dependence was analyzed within the dirty- and the clean-limit approaches. In the dirty-limit theory $\lambda^{-2}(T)$ has the form ${ }^{31}$

$$
\left.\frac{\lambda^{-2}(T)}{\lambda^{-2}(0)}\right|_{\text {dirty }}=\frac{\Delta(T)}{\Delta(0)} \tanh \left[\frac{\Delta(T)}{2 k_{B} T}\right],
$$

while in the clean limit, ${ }^{31}$

$$
\left.\frac{\lambda^{-2}(T)}{\lambda^{-2}(0)}\right|_{\text {clean }}=1+2 \int_{\Delta(T)}^{\infty}\left(\frac{\partial f}{\partial E}\right) \frac{E}{\sqrt{E^{2}-\Delta(T)^{2}}} d E .
$$

Here $f=\left[1+\exp \left(E / k_{B} T\right)\right]^{-1}$ is the Fermi function. The temperature dependence of the gap was approximated by $\Delta(T)$ $=\Delta(0) \tanh \left\{1.82\left[1.018\left(T_{c} / T-1\right)\right]^{0.51}\right\} .{ }^{14}$ As is seen, both the dirty- and the clean-limit approaches describe the experimental data reasonably well. The fitted curves are almost indistinguishable from each other. The results of the fits are $T_{c}$ $=2.11(2) \mathrm{K}, \Delta(0)=0.35(1) \mathrm{meV}$, and $2.12(2) \mathrm{K}$ and $0.39(1)$ $\mathrm{meV}$ for the dirty- and the clean-limit BCS model, respectively. The corresponding gap-to- $T_{c}$ ratios were found to be $\left[2 \Delta(0) / k_{B} T_{c}\right]_{\text {dirty }}=3.83(10)$ and $\left[2 \Delta(0) / k_{B} T_{c}\right]_{\text {clean }}=4.27(11)$. There are few reasons why, we believe, $\mathrm{Mo}_{3} \mathrm{Sb}_{7}$ studied here is in the dirty limit: (i) The maximum gap value obtained in Andreev-reflection experiments was found to be $\Delta(0)$ $\simeq 0.32 \mathrm{meV}$, which is close to $\Delta(0)=0.35(1) \mathrm{meV}$ observed within our dirty-limit calculations. (ii) The residual resistivity ratio measured on similar single crystals was found to be rather low $\rho_{300 \mathrm{~K}} / \rho_{0 \mathrm{~K}}=1.4{ }^{3}$ Note that the isostructural compound $\mathrm{Ru}_{3} \mathrm{Sn}_{7}$ has $\rho_{300 \mathrm{~K}} / \rho_{0 \mathrm{~K}}=144,{ }^{32}$ which is bigger by more than 2 orders of magnitude. (iii) A reasonable assumption about Fermi velocity $v_{F} \simeq 10^{6} \mathrm{~m} / \mathrm{s}$ can be made within the free-electron approximation by taking $E_{F} \simeq 6.5 \mathrm{eV} .{ }^{33}$ This allows us to readily estimate the BCS-Pippard coherence length $\xi_{0}=\hbar v_{F} / \pi \Delta(0) \simeq 600 \mathrm{~nm}$, which is approximately 50 times bigger than $\xi=13(1) \mathrm{nm}$ obtained experimentally. Considering that $\xi^{-1}=\xi_{0}{ }^{1}+l^{-1}(l$ is the mean free path), we obtain that the coherence length $\xi$ in $\mathrm{Mo}_{3} \mathrm{Sb}_{7}$ is limited by $l$ and, correspondingly, $l \simeq \xi \simeq 13 \mathrm{~nm}$.

Now we are going to comment shortly on the results of the specific heat experiments of Tran et al. ${ }^{6}$ It was shown that the analysis of the electronic specific-heat data within the framework of the phenomenological alpha model suggests the presence of two gaps with $2 \Delta_{1}(0) / k_{B} T_{c}=4.0$ and $2 \Delta_{2}(0) / k_{B} T_{c}=2.5$, and the relative weight of the bigger gap of 0.7. ${ }^{6}$ The $\lambda^{-2}(T)$ simulated by using these parameters and $\lambda(0)=716 \mathrm{~nm}$ is shown in Fig. 4 by blue dashed line. Both contributions were assumed to be in the dirty limit [see Eq. (4)] and the similar alpha model, but adapted for calculation of the superfluid density, was used. ${ }^{14,15}$ The agreement between the simulated curve and the experimental data is rather good. We should emphasize, however, that in the two-gap superconductor the contribution of the smaller gap to the total superfluid density decreases very fast with increasing field, thus leading to strong suppression of $\lambda^{-2} \cdot{ }^{15,25,26}$ This is inconsistent with the data presented in the inset of Fig. 2, revealing that in $\mathrm{Mo}_{3} \mathrm{Sb}_{7} \lambda^{-2}(B)=$ const.

To conclude, the superconductor $\mathrm{Mo}_{3} \mathrm{Sb}_{7}\left(T_{c} \simeq 2.1 \mathrm{~K}\right)$ was studied by means of muon-spin rotation. The main results are: (i) The absolute values of the magnetic-field penetration depth $\lambda$, the Ginzburg-Landau parameter $\kappa$, and the first $H_{c 1}$ and the second $H_{c 2}$ critical fields at $T=0$ were found to be $\lambda(0)=720(100) \mathrm{nm}, \kappa(0)=55(10), \mu_{0} H_{c 1}(0)$ $=1.8(3) \mathrm{mT}$, and $\mu_{0} H_{c 2}(0)=1.9(2) \mathrm{T}$. (ii) Over the whole temperature range (from $T_{c}$ down to $20 \mathrm{mK}$ ) the temperature dependence of $\lambda^{-2}$ is consistent with what is expected for a single-gap $s$-wave BCS superconductor. (iii) The ratio $2 \Delta(0) / k_{B} T_{c}=3.83(10)$ was found, suggesting that $\mathrm{Mo}_{3} \mathrm{Sb}_{7}$ superconductor is in the intermediate-coupling regime. (iv) The magnetic penetration depth $\lambda$ is field independent, in agreement with what is expected for a superconductor with a single isotropic energy gap. (v) The electronic mean free path was estimated to be $l \simeq 13 \mathrm{~nm}$. This relatively short value suggests that strong scattering processes play an important role in the electronic properties of $\mathrm{Mo}_{3} \mathrm{Sb}_{7}$. 
This work was performed at the Swiss Muon Source $(\mathrm{S} \mu \mathrm{S})$, Paul Scherrer Institute (PSI, Switzerland). The authors are grateful to A. Amato and D. Herlach for providing the instrumental support during the $\mu \mathrm{SR}$ experi- ments, and A. Maisuradze for calculating $\sigma_{s c}(b)$ dependences. The work was supported by the K. Alex Müller Foundation and in part by the SCOPES Grant No. IB7420110784. *rustem.khasanov@psi.ch

${ }^{1}$ P. Jensen and A. Kjekshus, Acta Chem. Scand. (1947-1973) 20, 417 (1966).

${ }^{2}$ F. Hulliger, Nature (London) 209, 500 (1966).

${ }^{3}$ Z. Bukowski, D. Badurski, J. Stepien-Damm, and R. Troć, Solid State Commun. 123, 283 (2002).

${ }^{4}$ C. Candolfi, B. Lenoir, A. Dauscher, C. Bellouard, J. Hejtmánek, E. Šantavá, and J. Tobola, Phys. Rev. Lett. 99, 037006 (2007).

${ }^{5}$ C. Candolfi, B. Lenoir, A. Dauscher, J. Hejtmánek, E. Šantavá, and J. Tobola, Phys. Rev. B 77, 092509 (2008).

${ }^{6}$ V. H. Tran, W. Miiller, and Z. Bukowski, arXiv:0803.2948 (unpublished).

${ }^{7}$ V. M. Dmitriev, L. F. Rybaltchenko, L. A. Ishchenko, E. V. Khristenko, Z. Bukowski, and R. Troć, Supercond. Sci. Technol. 19, 573 (2006).

${ }^{8}$ V. M. Dmitriev, L. F. Rybaltchenko, E. V. Khristenko, L. A. Ishchenko, Z. Bukowski, and R. Troć, Low Temp. Phys. 33, 295 (2007).

${ }^{9}$ V. M. Dmitriev, L. F. Rybaltchenko, L. A. Ishchenko, E. V. Khristenko, Z. Bukowski, and R. Troć, Low Temp. Phys. 33, 1009 (2007).

${ }^{10}$ R. Khasanov, D. G. Eshchenko, D. Di Castro, A. Shengelaya, F. La Mattina, A. Maisuradze, C. Baines, H. Luetkens, J. Karpinski, S. M. Kazakov, and H. Keller, Phys. Rev. B 72, 104504 (2005).

${ }^{11}$ R. Prozorov and R. W. Giannetta, Supercond. Sci. Technol. 19, R41 (2006).

${ }^{12}$ W. N. Hardy, D. A. Bonn, D. C. Morgan, R. Liang, and K. Zhang, Phys. Rev. Lett. 70, 3999 (1993).

${ }^{13}$ R. Kadono, J. Phys.: Condens. Matter 16, S4421 (2004).

${ }^{14}$ A. Carrington and F. Manzano, Physica C 385, 205 (2003).

${ }^{15}$ R. Khasanov, A. Shengelaya, A. Maisuradze, F. La Mattina, A. Bussmann-Holder, H. Keller, and K. A. Müller, Phys. Rev. Lett. 98, 057007 (2007).

${ }^{16}$ R. Khasanov, S. Strässle, D. Di Castro, T. Masui, S. Miyasaka, S. Tajima, A. Bussmann-Holder, and H. Keller, Phys. Rev. Lett. 99, 237601 (2007).

${ }^{17}$ E. H. Brandt, Phys. Rev. B 37, 2349 (1988).
${ }^{18}$ E. H. Brandt, Phys. Rev. B 68, 054506 (2003).

${ }^{19}$ D. T. Adroja, A. D. Hillier, J. G. Park, E. A. Goremychkin, K. A. McEwen, N. Takeda, R. Osborn, B. D. Rainford, and R. M. Ibberson, Phys. Rev. B 72, 184503 (2005).

${ }^{20}$ M. H. S. Amin, M. Franz, and I. Affleck, Phys. Rev. Lett. 84, 5864 (2000).

${ }^{21}$ I. L. Landau and H. Keller, Physica C 466, 131 (2007).

${ }^{22}$ J. Sonier, J. Brewer, and R. Kiefl, Rev. Mod. Phys. 72, 769 (2000).

${ }^{23}$ R. Khasanov, A. Shengelaya, D. Di Castro, D. G. Eshchenko, I. M. Savić, K. Conder, E. Pomjakushina, J. Karpinski, S. Kazakov, and H. Keller, Phys. Rev. B 75, 060505(R) (2007).

${ }^{24}$ J. D. Fletcher, A. Carrington, P. Diener, P. Rodiere, J. P. Brison, R. Prozorov, T. Olheiser, and R. W. Giannetta, Phys. Rev. Lett. 98, 057003 (2007).

${ }^{25}$ S. Serventi, G. Allodi, R. De Renzi, G. Guidi, L. Romano, P. Manfrinetti, A. Palenzona, Ch. Niedermayer, A. Amato, and Ch. Baines, Phys. Rev. Lett. 93, 217003 (2004).

${ }^{26}$ M. Angst, D. Di Castro, D. G. Eshchenko, R. Khasanov, S. Kohout, I. M. Savić, A. Shengelaya, S. L. Budko, P. C. Canfield, J. Jun, J. Karpinski, S. M. Kazakov, R. A. Ribeiro, and H. Keller, Phys. Rev. B 70, 224513 (2004).

${ }^{27}$ E. H. Brandt, J. Low Temp. Phys. 73, 355 (1988).

${ }^{28}$ J. E. Sonier, R. F. Kiefl, J. H. Brewer, D. A. Bonn, J. F. Carolan, K. H. Chow, P. Dosanjh, W. N. Hardy, R. Liang, W. A. MacFarlane, P. Mendels, G. D. Morris, T. M. Riseman, and J. W. Schneider, Phys. Rev. Lett. 72, 744 (1994).

${ }^{29}$ A. Maisuradze, R. Khasanov, A. Shenegelaya, and H. Keller, arXiv:0805.3646 (unpublished).

${ }^{30}$ N. R. Werthamer, E. Helfand, and P. C. Hohenberg, Phys. Rev. 147, 295 (1966).

${ }^{31}$ M. Tinkham, Introduction to Superconductivity (Krieger, Malabar, FL, 1975).

${ }^{32}$ B. C. Chakoumakos and D. Mandrus, J. Alloys Compd. 281, 157 (1998).

${ }^{33}$ E. Dashjav, A. Szczepenowska, and H. Kleinke, J. Mater. Chem. 12, 345 (2002). 